\title{
A practical method for assessing reinforced concrete members under impact
}

\author{
N. Madjlessi \& D. M. Cotsovos \\ Institute of Infrastructure and Environment, Heriot-Watt University, UK
}

\begin{abstract}
The effect of the loading-rate on the dynamic response of reinforced concrete members under impact loading is investigated numerically through the use of three-dimensional dynamic nonlinear finite element analysis. The package employed is capable of realistically accounting for the triaxiality and the brittle nature characterising concrete material behaviour as well as the characteristics of the problem at hand, a wave propagation problem within a highly nonlinear medium. Due to the availability of tests data, the present study focusses on investigating the effect of impact loading on the behaviour of reinforced concrete beam specimens. The numerical predictions obtained provide detailed insight into the mechanisms underlying RC structural response and offer a quantitative description of the effect of loading-rate on certain important aspects of the exhibited behaviour. Based on the numerical predictions obtained, a physical model is proposed which is capable of realistically describing the behaviour of the $\mathrm{RC}$ structural elements under high rates of concentrated loading. The proposed physical model links the observed shift in structural response to the localised experimentally established and/or numerically predicted behaviour with increasing rates of applied loading. Its formulation is based on the use of the Compressive Force Path method which is capable of realistically describing the behaviour of a wide range of reinforced concrete structural configurations at their ultimate limit state under both static and seismic loading conditions.

Keywords: reinforced concrete, impact, loading rate, compressive force path method, finite elements, nonlinear dynamic analysis.
\end{abstract}

\section{Introduction}

Impact loads are applied locally during the collision of an object onto a certain area of a structure. Their form (intensity, distribution, duration, loading-rate, time- 
history) depends on the mass, velocity and shape of the impacting object, the dynamic characteristics of the structural element considered (mass, stiffness) and the properties of the contact area. Available numerical and test data [1-6] reveal that with increasing loading rates RC structural response (observed through the deflection and cracking profiles exhibited) becomes more localised, since the portion of the $\mathrm{RC}$ element reacting to the external load reduces in length (concentrating around the impact region) (Fig. 1) as failure occurs prior to the generated waves reaching the supports $[5,6]$. This phenomenon, combined with the inertia forces developing along the element span in the transverse direction, underlie the mechanisms governing RC structural response [5-7].
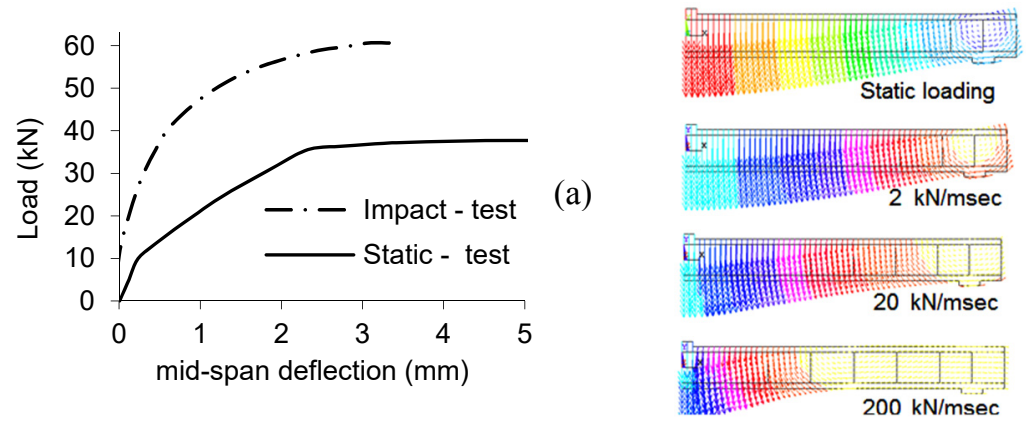

Figure 1: (a) Load-deflection curves [2] accompanied by (b) deformation profiles exhibited by RC beams under static and impact loading [5].

The experimental and numerical methods available for studying in detail RC structural response under impact loading are characterised by a certain degree of complexity and the predictions obtained by a certain level of uncertainty. In an attempt to predict RC structural response under impact in a practical way Single Degree of Freedom (SDOF) models have been developed [7, 8] which rely on available physical models, such as the truss analogy (TA), to describe the mechanism underlying the RC structural response approaching the ultimate limit state (ULS). The latter simplified method bypasses the complexities associated with the available experimental and numerical assessment methods, however, it fails to accurately account for the nature of the problem at hand (a wave propagation problem within a highly nonlinear material), the true mechanisms underlying $\mathrm{RC}$ structural response as well as the triaxiality and the brittleness characterizing concrete material behavior $[10,11]$. In an attempt to address this problem, a model is proposed herein which is based on the concepts underlying the 'Compressive Force Path' (CFP) method [10] in combination with a simplified assessment method which links the shift in structural behaviour observed under increasing loading rates with the shortening of the span of the $\mathrm{RC}$ beam reacting to the applied load [5-7]. This localized response can justify the observed increase in maximum sustained load and stiffness (see Fig. 1). The validation of the proposed model is achieved by comparing its predictions with its counterparts 
established experimentally, via drop-weight testing, and numerically, through the use of nonlinear finite element analysis (NLFEA).

\section{Limitations of available assessment methods}

Experimental methods: A large number of drop-weight tests [1-4] have been carried out to date mainly on individual RC elements (beams, columns, slabs). Such tests are difficult to conduct as the intensity of the loads generated increases rapidly (in a few msec) from zero to a maximum value often leading to explosive brittle forms of failure which can in turn damage the instruments employed for measuring structural response. Data obtained from such tests is characterised by considerable scatter partly due to a wide range of parameters (associated with the experimental setup and the specimen) which differ from test to test [5-7]. This scatter predominantly reflects the difficulty in correlating the measured response to the actual physical state of the specimens; in fact, the measured maximum value of imposed load frequently corresponds to a specimen physical-state characterised by high concrete disintegration as well as low residual load-bearing capacity and stiffness $[5,7]$. This stage of structural response has little practical significance as it depends heavily on post-failure mechanisms for transferring the applied loads to the specimen supports. In view of the above, the available test data cannot provide detailed insight into the mechanisms underlying RC structural response.

Detailed NLFEA: NLFEA is used as a safer and more efficient method for investigating a wider range of RC structural forms. It is capable of providing more detailed insight on the mechanisms underlying RC structural response under highrate loading compared to drop-weight testing. However, as it usually employs dense 3D finite element meshes, combined with complex constitutive material laws implemented through the use of iterative solution strategies, the required computational resources are high. As a result, its use is generally limited to the analysis of relatively simple structural forms. Moreover, its ability for providing realistic predictions of $\mathrm{RC}$ structural behaviour is, in most cases, linked with the use of case-study dependent constitutive models often incorporating empirical amplification factors to account for the effect of strain-rate sensitivity on concrete material behaviour [11].

Methods employed in practice: To avoid the complications and uncertainties associated with the previous assessment methods (mainly employed in research) and in order to simplify the analysis and design procedures, many (mainly military) design codes [9] employ equivalent simple lumped mass-spring systems for modelling individual structural elements with distributed mass and loading [8, 9]. The equivalence is based upon energy approximations that rely on an assumed deflected shape (the first eigenvector or the deflected shape under equivalent static loading). The latter methodology relies on a number of simplifications/ assumptions concerning both material behaviour and structural response. These include the use of simple uniaxial material laws, the description of post-failure behaviour, empirical amplification factors attributed to the strain-rate sensitivity of concrete behaviour, assumptions concerning the deformed shape of the structural elements and the use of elastic or elasto-plastic laws for describing 
structural behaviour. However, such simplifications do not allow the methodology to account for the brittle nature of concrete and its sensitivity to triaxial stress conditions, the true mechanics governing RC structural response as well as the localised response often exhibited.

\section{Physical models describing RC behaviour at ULS}

Unlike the TA method, which forms the basis of current design codes for RC structures (EC2, ACI), the CFP method [10] assumes that an RC structural element at its ULS behaves essentially as an arch-like structure (for the case of a simple supported beam) or a system of arch-like structures connected at the point of contraflexure (in the case of more intricate structural configurations characterised by static indeterminacy). The latter type of behaviour is enforced by the available reinforcement. The CFP method [10] accounts for the brittle nature and triaxiality characterising concrete material behaviour. It assumes that the area of the compressive zone has a significant effect on shear capacity while the contribution of aggregate interlock and dowel action is ignored. Finally, it is considered that under cyclic loading the inclined struts of the TA cannot form due to the densely spaced inclined intersecting cracks on the web of the $\mathrm{RC}$ element. The latter assumptions lead to a fundamentally different physical model for describing RC structural behaviour at the ULS. Failure is considered to occur due to the development of transverse tensile stresses at specific locations along the path followed by the compressive force. These locations are dependent on the value of the shear span-to-depth ratio $\left(a_{v} / d\right)$. The manner in which $a_{v} / d$ affects the loadcarrying capacity (expressed as $M_{u} / M_{f} ; M_{u}$ and $M_{f}$ are the of bending moments associated with failure and flexural capacity respectively) is indicated in Fig. 2 [10] in which four distinct types of structural behaviour are identified.

Type I behaviour is characterized by a flexural mode of failure preceded by longitudinal splitting of the concrete in the compressive zone of the beam. This occurs when concrete strength in the compressive zone is exhausted due to the development of transverse tensile stresses induced by volume dilation of concrete in the adjacent regions which include primary flexural cracks. This allows the maximum stresses developing within the compressive zone of the beam to attain values approximately equal to 1.5 times the uniaxial compressive strength of concrete $f_{c}[10]$.

Type II behavior is characterised by a brittle mode of failure usually caused by tensile stresses developing either in the region of change of the CFP direction (location 1 in Fig. 3(a)) or in the region of the cross-section where the maximum bending moment combines with the shear force (location 2 in Fig. 3(a)). The transverse stress resultant at location 1 is considered numerically equal to the acting shear force, and, its effect is considered to spread over a distance $d$, on either side of location 1. The value of the tensile force that can be sustained at this location is determined by eq. (1) [10].

$$
T_{I I, 1}=0.5 \cdot b \cdot d \cdot f_{t}
$$

$b$ and $d$ are the width and effective depth of the beam cross-section whereas $f_{t}$ is the tensile strength of concrete. 


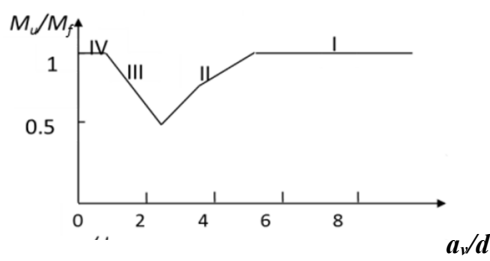

$\begin{array}{cc}\text { Type I } & a_{v} / d>\sim 5 \\ \text { Type II } & 2.5<a_{v} / d<\sim 5 \\ \text { Type III } & 1<a_{v} / d<2.5 \\ \text { Type IV } & a_{v} / d<1\end{array}$

Figure 2: Effect of $a_{v} / d$ on the load-carrying capacity $\left(M_{u} / M_{f}\right)$ of RC beams [10].
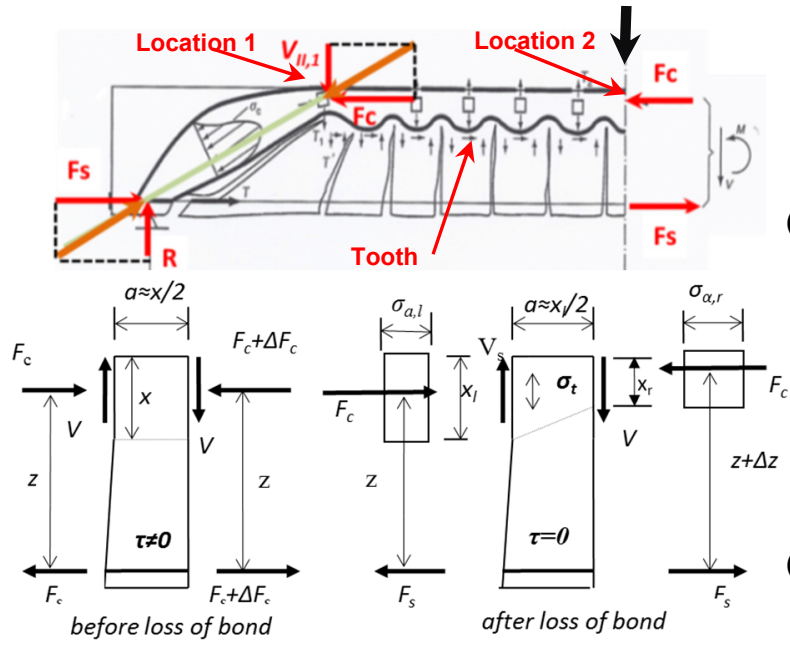

Figure 3: Internal actions acting: (a) on a RC beam exhibiting type II behaviour and (b) on a concrete tooth before and after loss of bond [9].

If the developing shear force is higher than the value provided by eq. (1), stirrups are uniformly placed over a length $d$ on both sides of location 1 in a quantity sufficient to sustain the whole value $\left(V_{f}\right)$ of the shear force corresponding to flexural capacity. The amount of stirrups required is provided by eq. (2) and their spacing $(s)$ should not exceed $0.5 d$.

$$
V_{f}=A_{s w, I I 1} f_{y w}(2 d / s+1)
$$

where $A_{s w, I I 1}$ is one stirrup's cross section.

Further to location 1, transverse tensile stresses within the compressive zone may also develop at location 2 (Fig. 3(a)) due to the loss of bond between the longitudinal reinforcement and the surrounding concrete. Fig. 3(b) indicates a portion of the beam between two cross-sections defined by consecutive cracks (concrete tooth), with the internal forces which develop at these cross-sections before and after the loss of bond. Based on the equations of equilibrium, the bending moment and shear forces are given by eqs (3) and (4) respectively.

$$
\begin{gathered}
M=F s^{\cdot} Z \\
V=d M / d x=d F s / d x^{\cdot} \cdot z+F s^{\cdot}(d z / d x)
\end{gathered}
$$

Loss of bond can lead to an extension of the right-hand crack and, hence, to a reduction of the depth of the compressive zone (see Fig. 3(b)).

$$
F_{c} \cdot\left(x_{l}-x_{r}\right) / 2=V \cdot x_{l} / 2
$$


This reduction leads to an increase of the intensity of the compressive stress field thus leading to dilation of the volume of concrete in the compressive zone, which in turn causes the development of transverse tensile stresses $\left(\sigma_{t}\right.$, see Fig. 3(b)) in the adjacent regions eq. (6).

$$
\left|\sigma_{t}\right|=f_{c} /\left[5\left(f_{y k} A_{s} / V-1\right)\right]
$$

By considering these transverse tensile stresses and the ensuing complex triaxial stress state it is possible to express the shear force $\left(V_{I I, 2}\right)$ that can be sustained at locations 2 form eq. (7) [10].

$$
V_{I I, 2}=F_{c} \cdot\left[1-1 /\left(1+5 \cdot f_{t} / f_{c}\right)\right]
$$

If the developing shear force is higher than the value provided by eq. (6), stirrups are needed. To calculate the amount of stirrups required it is necessary to calculate the vertical and the horizontal stress resultants in the region between (i) the area where the inclined and the horizontal portion of the compressive path meet (region 1, Fig. 4(a)) and (ii) the point at which the load is applied.

$$
\begin{aligned}
T_{I I, 2 v} & =\sigma_{t} b\left(\alpha_{v}-2 d\right) / 2 \\
T_{I I, 2 h} & =\sigma_{t} x\left(\alpha_{v}-2 d\right) / 2
\end{aligned}
$$

Based on Eqs (8) and (9), the amount of stirrups required is obtained from Eqs (10) and (11):

$$
\begin{aligned}
A_{s w, I I 2 v} & =T_{I I, 2 v} / f_{y w} \\
A_{s w, I I 2 h} & =T_{I I, 2 h} / f_{y w}
\end{aligned}
$$

Type III behavior, for which location 1 coincides with location 2 (i.e. the compressive zone into the shear span degenerates into a cross section within the shear span) is characterised by a brittle mode of failure caused by the deep penetration of the inclined crack into the compressive zone of the beam. This crack reduces the strength of the uncracked concrete in the compressive zone on the region where the inclined and the horizontal compressive path of the model meet (region 1, Fig. 3(a)), which causes a reduction on the flexural capacity of the beam. Based on the internal actions presented in Fig. 4(a), a measure of the maximum shear force that concrete alone can carry on this region is provided by eq. (12).

$$
V_{I I I}=M_{I I I} / a_{v}
$$

where: $M_{I I I}=M_{I I}^{(2.5 d)}+\frac{\left(M_{f}-M_{I I}^{(2.5 d)}\right)\left(2.5 d-a_{v}\right)}{(1.5 d)} \quad$ and $\quad M_{I I}^{(2.5 d)}=2.5 d V_{I I, 1}$

The stirrups required are provided by eq. (13) and are distributed within the shear span with a spacing $s$ smaller than $0.5 d$ :

$$
A_{s w, I I I}=2\left(M_{f}-M_{I I I}\right) /\left(a_{v} f_{y w}\right)
$$

Type IV behavior can be characterised by two modes of failure linked with either failure of the horizontal element of the CFP model or failure of the uncracked end portion of the beam (inclined leg of the 'frame' of the CFP model) in compression. From the moment equilibrium of the free body in Fig. 4(b), the flexural capacity $\left(M_{f}\right)$ can be easily calculated and consequently the associated load-carrying capacity $\left(P_{f}\right)$ can be determined from eq. (14).

$$
P_{f}=M_{f} / a_{v}
$$



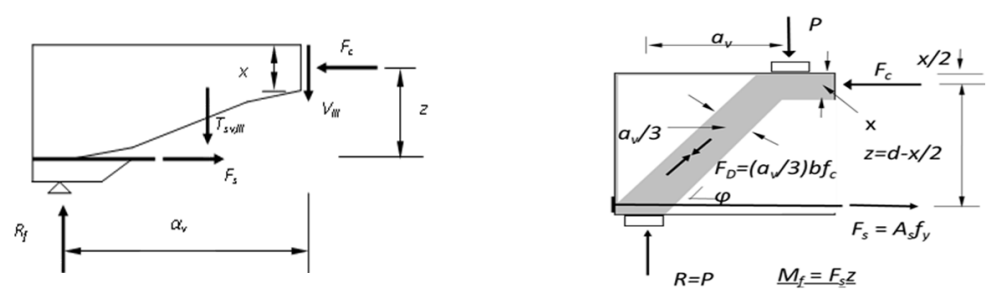

Figure 4: Internal actions developing in a RC beam exhibiting (a) type III and (b) type IV behaviour.

On the other hand, the load-carrying capacity $\left(P_{D}\right)$ corresponding to the strength of the inclined leg of the 'frame' will be equal to the vertical component of the load $\left(F_{D}\right)$ that can be carried by this leg. As indicated in Fig. 4(b), $F_{D}$ is easily calculated by taking the depth of the leg equal to $a_{v} / 3$ as recommended in [9].

$$
P_{D}=F_{D} z /\left(z+\alpha_{v}\right) l / 2 \text { where } F_{D}=\left(\alpha_{V} / 3\right) b f_{c}
$$

Therefore, the load-carrying capacity of a beam in the case of type IV behavior will be:

$$
P_{u}=\min \left(P_{F}, P_{D}\right)
$$

\section{Effect of loading rate on the behaviour of RC beams}

A schematic representation of the inertia forces resisting the action of an impact load exerted at the mid span section of a simple supported RC beam is shown in Fig. 5(a), together with the corresponding shear force (Fig. 5(b)) and bending moment (Fig. 5(c)) diagrams which helped to identify the portion of the RC beam's span $\left(L_{e f f}\right)$ which essentially reacts to the imposed impact load (Fig. 5(d)) [7]. This portion is assumed to be fully or partially fixed at its ends and can be used to form an equivalent static problem. Figure 5(e) shows the proposed physical model describing the mechanics underlying the response of the RC beam under increasing rates of loading on the basis of the equivalent static problem (Fig. 5(d)).

$L_{\text {eff }}$ is essentially the distance covered by the stress waves generated during impact within the time period $\Delta t_{c}$ within which cracks form at the upper face of the end sections of $L_{\text {eff }}$ (see Fig. 7(b)) [5-7]; hence,

$$
L_{\text {eff }}=2 \cdot v_{w} \cdot \Delta t_{c}
$$

with $v_{w}=\sqrt{ }(G / \rho)$ being an estimate of the velocity at which the stress wave travels within the concrete medium, where $G=E /(2+2 v)$ is the shear modulus, $E$ the modulus of elasticity, $v$ the Poisson's ratio and $\rho$ the density of concrete.

If $\dot{P}$ is the loading rate and considering the beam portion $L_{e f f}$ in Fig. $5(\mathrm{~d})$, then the value of the imposed load at which cracking forms at the upper face of the $L_{\text {eff }}$ ends is $P_{d, c r}=\dot{P} \cdot \Delta t_{c}=8 M_{c r} /\left(\alpha L_{e f f}\right)$. Replacing in the latter equation $L_{\text {eff }}$ as expressed in eq. (17) and solving in respect to $\Delta \mathrm{t}_{\mathrm{c}}$ results:

$$
\Delta t_{c}=\left[4 M_{c r} /\left(\alpha \dot{P} v_{w}\right)\right] 0.5
$$


Substituting the value of $\Delta t_{c}$ obtained from eq. (18) into eq. (17) provides the value of $L_{\text {eff. }}$. Considering different degrees of fixity being achieved at the ends of the

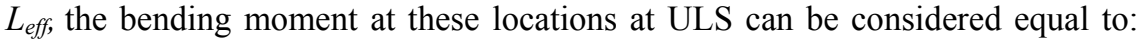
(i) the flexural capacity $M_{F_{2}}$ (fully fixed support) or (ii) the bending moment $M_{C R_{2}}$ at which cracking initiates at the upper face of the $L_{e f f}$ ends (see Fig. 6(b)). Based on the above assumptions the beam load-carrying capacity will be

$$
P_{F}=2 V_{F}
$$

where $V_{F}=(2 / a)\left(M_{F 1}+M_{F 2}\right) / L_{\text {eff }}(20 \mathrm{a}) \quad$ or $\quad V_{F}=(2 / a)\left(M_{F 1}+M_{C R 2}\right) / L_{\text {eff }}$

with $V_{F}$ being the resultant of the up-thrust forces (i.e. the resultant of the inertial forces $F_{I}$ and the reactions $R$, see Fig. 5) acting on $L_{\text {eff }}$ and $a$ a parameter defining the distance of $V_{F}$ from $P_{F}$ as a percentage of $L_{\text {eff }} / 2$ which may be assessed on the basis of the available experimental and numerical data as discussed in section 9 .

Equations (17) to (20) may form the basis of a simple method for assessing the beam load-carrying capacity under impact loading.

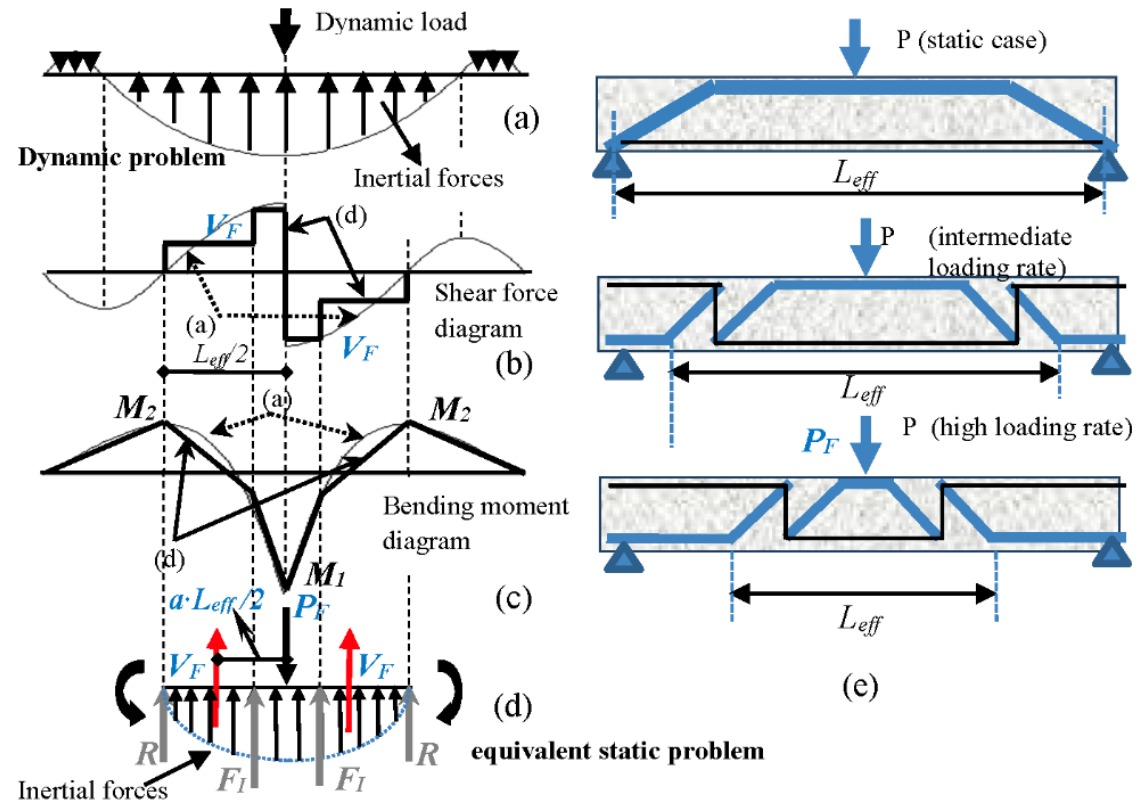

Figure 5: RC beam under impact loading: Schematic representations of (a) imposed loading, (b) shear force diagram, (c) bending moment diagram, (d) the equivalent static problem based on $L_{\text {eff }}$ and (e) the proposed physical model describing the behaviour of the RC beam under increasing loading rates.

\section{General aspects of the NLFEA model employed}

ADINA shares a number of characteristics with RCFINEL [11] which has been found capable of providing realistic predictions concerning the response of a wide 
range of $\mathrm{RC}$ structural configurations under static and dynamic loading. During each time step, the equation of motion is solved as a sequence of equivalent static problems through the use of the Newmark family of approximation methods. An iterative procedure based on the modified Newton-Raphson method is used for solving the equivalent static problem considered at each time increment. During each iteration, every Gauss point is checked to determine whether loading or unloading takes place and to establish whether any cracks close or form. Appropriate changes are then introduced to the stiffness matrix. Convergence is checked at each Gauss point; this involves the use of the constitutive relations for the calculation of the stresses and the corresponding strain increments. Once the values of the residual forces become smaller than a predefined value convergence is accomplished otherwise the residual forces are re-imposed onto the FE model until convergence is finally achieved.

The concrete material model employed by ADINA stems from experimental data obtained from tests conducted on concrete cylinders under triaxial loading conditions [11]. It is fully defined by a single material parameter - the uniaxial cylinder compressive strength $f_{c}$, and accounts for the brittle nature and triaxiality characterising concrete material behaviour. A simple bilinear elasto-plastic hardening model is employed for describing the behaviour of steel. Concrete and steel material behaviour is assumed to be independent of the loading-rate and full bond is considered between steel and concrete.The smeared-crack approach is adopted for modelling cracking. The development of a crack is followed by immediate loss of load-carrying capacity in the direction normal to the plane of the crack. At the same time, the shear stiffness is also reduced drastically to about $10 \%$ of its value before the occurrence of the crack. Each integration point can develop up to three cracks.

\section{Structural form investigated}

The behaviour of the RC beam specimens considered herein have been experimentally investigated in the past [1] under static and drop-weight testing. The design details of specimens C2 and D1 considered in the present work are presented in Fig. 6(a).The modulus of elasticity, yield stress and ultimate strength of all types of steel reinforcement are $E_{S}=206 \mathrm{GPa}, f_{y}=460 \mathrm{MPa}$ and $f_{u}=560$ $\mathrm{MPa}$, respectively. The uniaxial compressive strength $\left(f_{c}\right)$ of concrete is $45 \mathrm{MPa}$.

\section{FE modelling of the problem at hand}

Concrete is modelled using a mesh of 27-noded brick elements (which adopt a $3 \times 3 \times 3$ integration rule) with an edge size of approximately $40 \mathrm{~mm}$. The steel reinforcement bars are modelled as 2-node truss elements of appropriate crosssectional area. Due to the double symmetry of the problem at hand, only a quarter of the RC beam is modelled with suitable boundary conditions, see Fig. 5(b). The load is applied onto the mid-span of the beam through a steel plate. For the case of static loading the load is applied monotonically until failure in the form of 
displacement increments (displacement control). In the dynamic case studies the load is imposed with at a constant rate in the form of load increments.

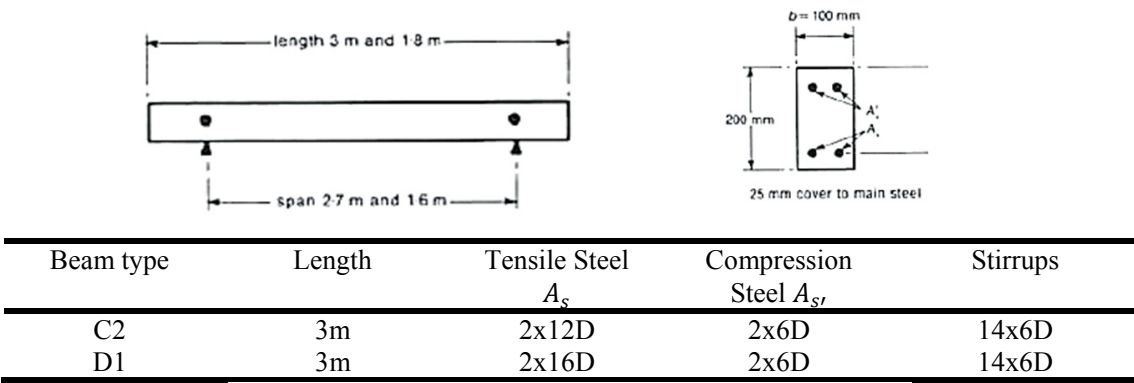

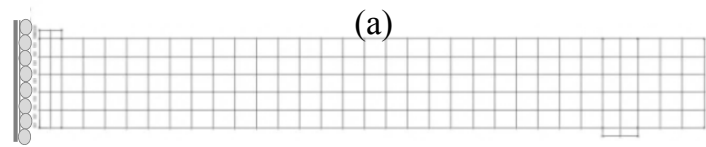

(b)

Figure 6: $\quad$ RC beams investigated: (a) Design details; (b) FE model employed.

\section{Numerical predictions}

Static case study: Under static loading, the predictions obtained concerning certain aspects of the behaviour exhibited by specimens $\mathrm{C} 2$ and D1 are presented in Fig. 7. The predictions in Fig. 7(a) are presented in the form of curves describing the relation between the applied load and the deflection at mid-span. The latter curves are in good agreement with their experimentally established counterparts [1]. Both, measured and predicted response show that all beams exhibited ductile behaviour, with failure occurring after yielding of the longitudinal reinforcement bars at mid-span, resulting in the formation of extensive cracking that led to loss of load-carrying capacity of the compressive zone. Fig. 7(b) shows the deformation and cracking profiles of specimen $\mathrm{C} 2$ at different stages of the loading process. Overall, the numerically predicted response of the $\mathrm{RC}$ beams is in agreement to that established experimentally.

High rate loading case studies: The values of the applied loading rates considered in the numerical study range from 1 to $10^{3} \mathrm{kN} / \mathrm{ms}$. From the predicted loaddeformation curves presented in Fig. 8(a), it appears that an increase in the loading rate leads to an increase in stiffness and load-carrying capacity combined with a decrease of the maximum deflection at mid span. As regards the cracking and deformation profiles, Fig. 8(b) indicates that, under relatively low rates of impact loading, beam behaviour is qualitatively similar to that exhibited under static loading. However, as the rate of loading increases, the portion $\left(L_{e f f}\right)$ of the beam mostly affected by the applied load reduces; for relatively high rates of loading, it is confined in the region of the beam mid-span extending on either side of the midspan cross section to a distance marked by the formation of vertical (flexural) cracking initiating at the upper face and extending downwards, whereas the remainder of the beam, i.e. the portions extending between the supports and the aforementioned cracking, practically remain unaffected by the applied load. 
Therefore, under high rates of loading, beam behaviour is essentially controlled by $L_{\text {eff. }}$. The variation of the dynamic increase factor (DIF), i.e., the increase in the maximum load sustained by the $\mathrm{RC}$ beams under high rate loading normalised with respect to its counterpart under static loading, with the applied loading rate is presented in Fig. 9 which shows a good correlation between the values predicted numerically and their experimental counterparts.
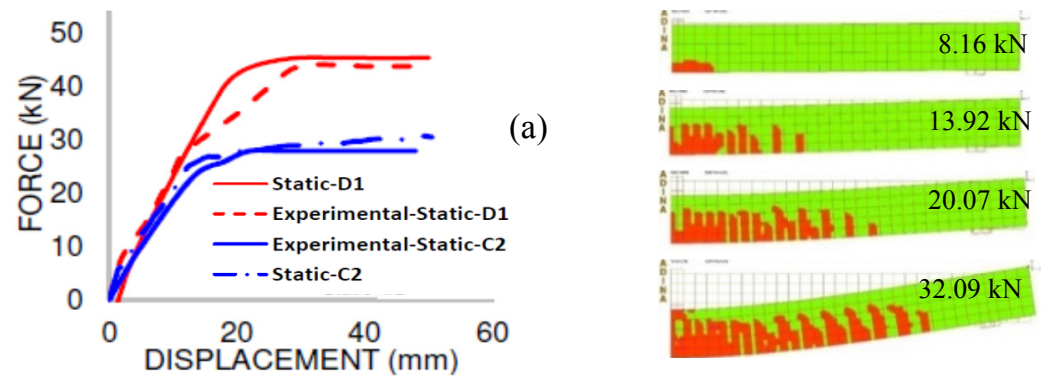

Figure 7: Beam C2 under static loading: (a) Experimentally established and numerically predicted load-deflection curves; (b) Numerically predicted deformation and cracking profiles at various load levels.
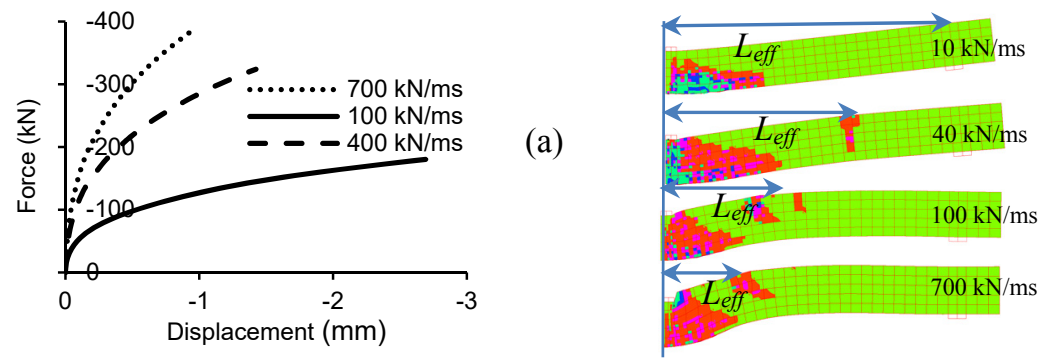

Figure 8: Beam C2 under loading applied at various rates: (a) Load-deflection curves; (b) Deformation and cracking profiles.
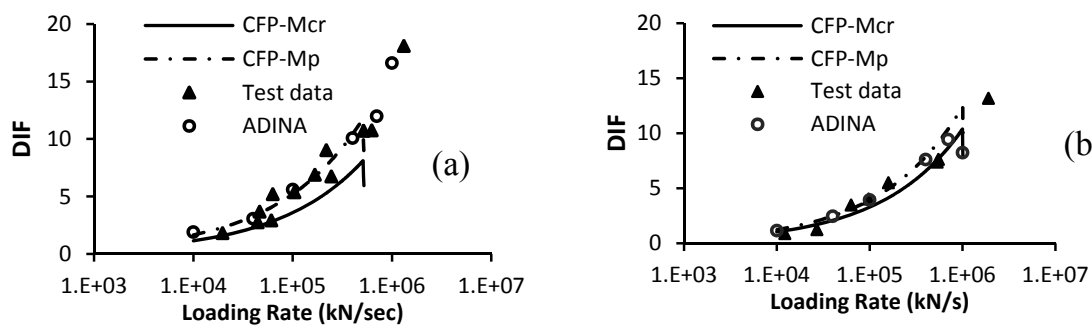

Figure 9: Variation of DIF with increasing loading rate: (a) beam $\mathrm{C} 2$ and (b) beam D1. 


\section{Calibration of the proposed method}

Substituting $a \approx 1$ into eqs (17)-(20) in Section 4 the predictions obtained by the simplified method appear to be in good agreement with both the experimental and numerical data up to a certain value of loading rate $\left(10^{6} \mathrm{kN} / \mathrm{msec}\right.$, see Fig. 9). Beyond this limit of loading rate structural response becomes too localised and as a result the simplified methodology proposed is no longer applicable. However, for loading rates up to $10^{6} \mathrm{kN} / \mathrm{msec}$ the proposed method appears capable of explaining how the reduction of the element span $\left(L_{e f f}\right)$ reacting to the imposed load can affect the mechanics underlying $\mathrm{RC}$ structural response when approaching ULS. The experimentally and numerically observed shift in structural response with increasing loading rates is essentially attributed to the change of the behaviour type exhibited by the span $\left(L_{\text {eff }}\right)$ reacting to the imposed load which gradually shifts form Type I to IV. The formulation of the present method is currently further refined by considering a wider range of tests data obtained from drop weight tests as well as additional predictions obtained from parametric studies conducted through the use of NLFEA.

\section{Concluding remarks}

The numerical predictions of the simplified assessment method are in good agreement with their counterparts obtained experimentally (via drop weight testing) and numerically (via dynamic nonlinear finite element analysis). The proposed physical model (which forms the basis of the simplified method) is capable of describing the response of RC beams when approaching the ULS under high rate loading. The proposed method is currently being refined and forms the basis for the development of a new SDOF model which will be capable of providing realistic predictions concerning the behaviour of individual RC structural elements under impact loading.

\section{References}

[1] Hughes G. Spiers D.M. (1982) "An investigation on the beam impact problem". Cement and Concrete Association, Technical Report 546.

[2] Miyamoto A., King M. W. Fujii M. (1989) "Non-linear dynamic analysis and design concepts for RC beams under impulsive loads". Bulletin of the New Zealand National Society for Earthquake Engineering, vol. 22, pp. 98111.

[3] Chen Y., May I. (2009) 'Reinforced Concrete members under drop-weight impact'. ICE Proc. Struct. \& Build. 162 (SB1), 45-56.

[4] Saatci S., Vecchio F. (2009) 'Effects of shear mechanisms on impact behavior of reinforced concrete beams', ACI Struct. J., 106(1), 78-86.

[5] Cotsovos D.M., Pavlović M.N. (2012) 'Modelling of RC Beams under Impact Loading'. ICE Proc. Struct. \& Build. 165(2): 77-94. 
[6] Cotsovos D.M., Stathopoulos N.D., Zeris Ch. (2008) 'Fundamental behaviour of RC beams subjected to high rates of concentrated loading'. J. of Struct. Engng., ASCE, 134, 1839-1851.

[7] Cotsovos D.M. (2010), 'A Simplified approach for assessing the loadcarrying capacity of reinforced concrete beams subjected to impact loading". Int. J. Imp. Engng, 37, 907-917.

[8] Morison CM (2006), 'Dynamic response of walls and slabs by singledegree-of-freedom analysis - a critical review and revision', Int. J. Impact Engng, 32, 1214-1247.

[9] TM5-855-1 (1998), Design and Analysis of Hardened Structures to Conventional Weapons Effects, Departments of Army, Air Force and Navy and the Defense Special Weapons Agency, USA.

[10] Kotsovos M.D. (2013) Compressive Force-Path Method Unified Ultimate Limit-State Design of Concrete Structures, Springer.

[11] Kotsovos M.D. (2015) Finite-Element Modelling of Structural Concrete: Short-Term Static and Dynamic Loading Conditions, CRC Press. 